\title{
SERIES INTRODUCTION \\ Heparan sulfate proteoglycans: intricate molecules with intriguing functions
}

\author{
Renato V. Iozzo \\ Department of Pathology, Anatomy and Cell Biology, and the Cellular Biology and Signaling Program, \\ Kimmel Cancer Center, Thomas Jefferson University, Philadelphia, Pennsylvania, USA \\ Address correspondence to: Renato V. Iozzo, Department of Pathology, Anatomy and Cell Biology, Room 249, \\ Jefferson Alumni Hall, Thomas Jefferson University, 1020 Locust Street, Philadelphia, Pennsylvania 19107, USA. \\ Phone: (215) 503-2208; Fax: (215) 923-7969; E-mail: iozzo@lac.jci.tju.edu. \\ J. Clin. Invest. 108:165-167 (2001). DOI:10.1172/JCI200113560.
}

The term heparan sulfate proteoglycan (HSPG) refers to a dichotomous structure, a protein coupled to a unique glycosaminoglycan chain characterized by a linear array of alternating disaccharide units. Both the protein core and the side chains undergo a series of modification reactions, which endow them with a very complex fabric (1). The final product is a sophisticated compound that can fluctuate in size, fine structure, and biology (2-5). The complexity of a usual heparan sulfate chain, for instance, can easily surpass that of common nucleic acids $(6,7)$.

The field of proteoglycan research is nearly equally split into two factions: those who feel that heparan sulfate is the prima donna, with the protein core being just a carrier, and those who feel that there would be no role for heparan sulfate without the protein core. The truth, as usual, is in the middle. Both possess unique features that in some respects act synergistically to provide the maximal strength of signaling and biological activity. This Perspective series, motivated by the recent flurry of discoveries, has been coordinated to provide a balanced view of the most recent advances in the field of HSPG research. The development of novel experimental strategies for decoding the sequence of heparan sulfate (8-10), together with the availability of antibodies specific for heparan sulfate epitopes (11), holds promise for deciphering the functional specificity of heparan sulfate in tissues. The medical importance is underscored by the direct involvement of HSPGs in various human diseases involving the musculoskeletal system, such as the Schwartz-Jampel chondrodystrophic myotonia (12) and the dyssegmental dysplasia Silverman-Handmaker type (13), overgrowth syndrome (Simpson-Golabi-Behmel) (14), or hereditary bone disorders (hereditary multiple exostoses) (15).

Functional complexity and structural plasticity The number of genes encoding "full-time" HSPGs is quite limited. Five distinct classes of cell surface and pericellular HSPGs (Figure 1), representing the prod- ucts of only 13 genes, probably account for at least $95 \%$ of the heparan sulfate of mammalian cell surfaces, basement membranes, and ECMs, while the remainder consists of several "part-time" HSPGs, such as betaglycan, CD44/epican, and testican. The cell-associated HSPGs include four integral membrane syndecans and six glycosyl-phosphatidylinositol-anchored (GPI-anchored) glypicans. The three pericellular HSPGs include one copy each of perlecan, agrin, and the hybrid HSPG/collagen type XVIII. Moreover, splice variants have also been reported for several protein cores, including perlecan, agrin, and type XVIII collagen.

All of these HSPGs are, in one way or another, affiliated with cells. Basement membranes are thin, cellassociated sheets of multimodular molecules, most of which interact with each other to form a barrier to charged molecules, thereby acting as true biological filters. The three HSPGs that do not have a direct link to the plasma membrane (perlecan, agrin, and collagen type XVIII) are also very closely associated with the cell plasma membrane, most likely bound via integrins and perhaps other, not yet identified receptors. Because integrin binding might be very powerful and cooperative in nature, the linkage to the plasma membrane might be indeed very strong. Thus, I prefer to call these HSPGs "pericellular" rather than "extracellular" proteoglycans. Regardless of the semantic distinction, all of them are truly cellular extensions into the immediate microenvironment, a sort of biological sensors of their surroundings and effectors within the extracellular milieu.

A few take-home messages

The heparan sulfate chains vary in length from $\sim 5$ to $\sim 70 \mathrm{kDa}$. This is an important and often forgotten point. Glycosaminoglycan chains, joined to the polypeptide, also enjoy ample freedom to move laterally. These linear polymers can reach distant targets that can be located several hundreds of nanometers 


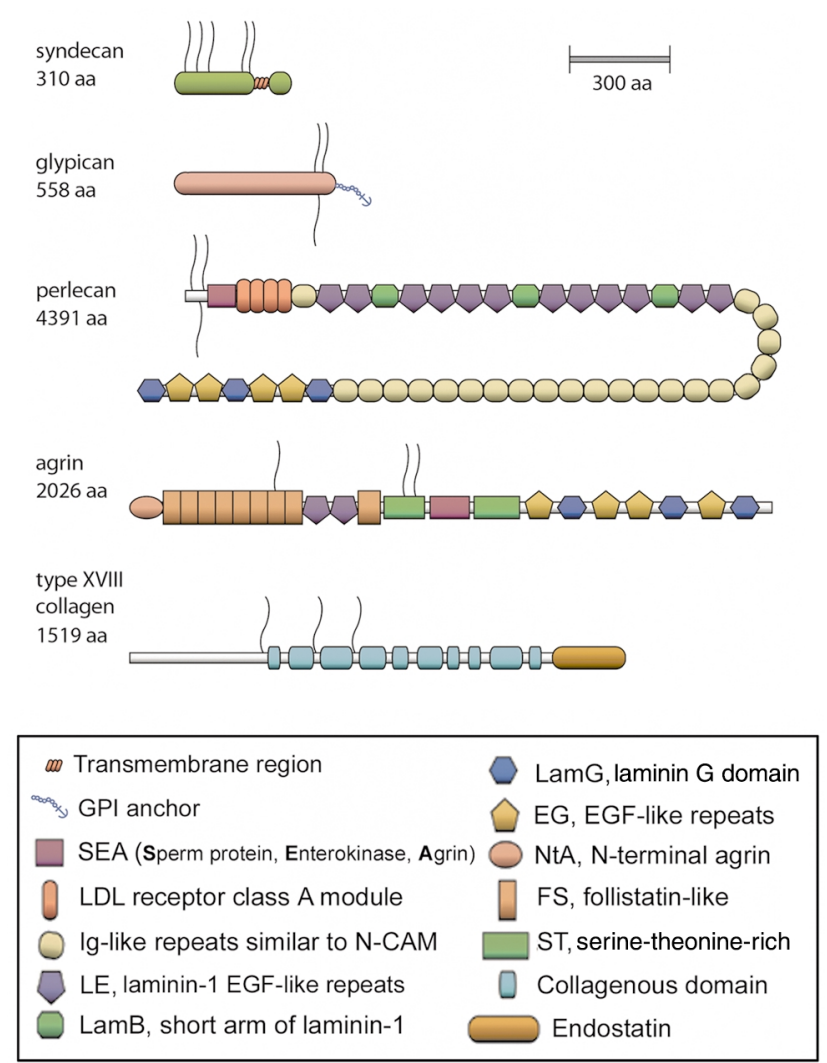

Figure 1

Schematic representation of the five HSPG prototypes. A total of thirteen distinct genes encompass nearly all the full-time HSPGs. These include four syndecans, six glypicans, and one each of perlecan, agrin, and the hybrid HSPG/collagen type XVIII. In addition, splice variants have been reported for perlecan, agrin, and type XVIII collagen. The size is roughly proportional to the number of amino acid residues (aa). The linear schemes do not take into account globular domains or other types of protein folding. The key to the various color-coded modules is provided in the bottom panel. The heparan sulfate chains are shown as wavy lines.

away. Thus, heparan sulfate could easily cross a fullthickness basement membrane and touch an adjacent cell. The protein cores vary in size from about 32 to nearly $500 \mathrm{kDa}$ (Figure 1). However, relatively few modules are used for building the protein core of various HSPGs, and even fewer for the covalent binding of the heparan sulfate chains. Diversity in protein core structure is, thus, a fraction of that expressed by heparan sulfate. Most, if not all, HSPGs can be hybrid molecules, carrying both heparan and chondroitin sulfate side chains. We do not know what tissue- and cell-specific constraints regulate the addition of these glycosaminoglycans or other aspects of the fate of a given HSPG, such as its targeting to a specific compartment within the membrane or its ability to be displaced by added heparin.

The series

The Perspectives in this and following issues update our understanding of HSPG biology in various areas of basic research and clinical medicine, encompassing cancer; angiogenesis; tumor invasion; hereditary disor- ders, both induced and uninduced; and viral diseases. Before considering the role of HSPGs in disease states, it is essential to have a good grasp of the biosynthetic pathways leading to the formation of the complex heparan sulfate. Esko and Lindahl provide an overview of heparan sulfate structure and biosynthesis and set the stage for discussing how alterations in this fascinating class of compounds can lead to human disorders. Understanding heparan structure and biosynthesis would also assist in understanding the potential implication of abrogating specific enzymes involved in its biosynthesis and modifications. The nearly mathematical perfection of heparan sulfate suggests that the biosynthetic enzymes that generate this structure are arranged in a rigorously controlled sequence. Forsberg and Kjellén provide an overview of various gene knockouts involved in the biosynthesis of either the heparan sulfate or specific protein cores. In both cases, phenotypes are often unpredictable and raise more questions.

Because of the enormous repertoire of interactions, Gallagher emphasizes the promiscuous role of heparan sulfate, focusing on the fine molecular structure that ultimately dictates its function, and on the interactions with growth factors and morphogens. Not only the heparan sulfate chains, but also bits and pieces of the protein cores, i.e., proteolytically processed isoforms, behave quite remarkably. Iozzo and San Antonio discuss the emerging role of HSPGs in angiogenesis. Some fragments bind angiogenic stimuli and seem to induce the formation of new blood vessels, while others behave as suppressors. Researchers have recently discovered a treasure-trove of these molecules, which are often small modules within a larger array of individual modules, often repeated multiple times. Several enzymes that tumor cells employ to invade the surrounding host tissues can also generate these bioactive modules, but often the tumor cells override the inhibition by generating angiogenesis stimulators. In addition, aggressive tumor cells can synthesize a variety of glycosidases. Vlodavsky and Friedmann address the story of heparanase, a unique mammalian enzyme whose abnormal expression correlates with an enhanced metastatic phenotype and augmented tumor angiogenesis. Regulation of heparanase occurs at many levels, including the subcellular localization of the enzyme, physical association with a given HSPG, membranebound proteases, local $\mathrm{pH}$, and cytokine expression.

The biosynthesis and degradation of heparan sulfate is under tight control mechanisms. Whereas the protein cores of HSPGs can be proteolytically processed or degraded by a variety of enzymes, only one mammalian heparanase appears to be responsible for digesting the heparan sulfate chains. Unlike the bacterial enzymes that act on heparan sulfate, which are classified as eliminases because they cleave the glycosaminoglycan from the protein core in toto, the mammalian heparanase is a hydrolase and cleaves between the saccharide subunits of the glycosaminoglycan chain. Heparanase activity is present in both endosomal and lysosomal compartments, but it can also occur at the cell surface, where it might digest adjacent HSPGs. Thus, the level of complexity in HSPG research is quite high. 
Like heparanase, the heparan sulfate biosynthetic machinery is also highly conserved, as shown by genetic studies in Drosophila that have implicated glypicans in signaling mediated by Wnt and TGF- $\beta$ families of growth factors. Filmus and Selleck overview this very interesting and clinically relevant field and discuss how glypicans are proteoglycans with a surprising involvement in insulin growth factor signaling, rather than FGF signaling as previously thought. Genetic defects in Glypican-3 lead to a human overgrowth syndrome, indicating that this proteoglycan functions as a negative growth regulator. Duncan, McCormick, and Tufaro review a very interesting field of bone biology in which it has come to light that heparan sulfate polymerases can serve as tumor suppressor genes. In the case of hereditary multiple exostoses, a dominantly inherited genetic disorder characterized by the formation of multiple cartilaginous tumors, extensive genetic analyses of affected families have linked mutations to two members of a novel family of putative tumor suppressor genes, EXT1 and EXT2. These genes code for enzymes, exostosin- 1 and exostosin-2, that polymerize heparan sulfate. The biological function of these proteins emerged in part by way of a serendipitous discovery made in the study of herpes simplex virology. Indeed, HSPGs have been implicated in a variety of viral disorders, mostly as coreceptors that facilitate entry of the virus. In the last Perspective, Shukla and Spear address the role of heparan sulfate in infection of cells by human members of the herpes virus family. Several of the human herpes viruses can make their initial contact with cells by binding to cell surface heparan sulfate. Eight human herpesviruses, which differ markedly in their biology, include herpes simplex types 1 and 2, varicella-zoster virus, cytomegalovirus, Epstein-Barr virus, human herpesvirus 6 , human herpesvirus 7 , and human herpesvirus 8 (or Kaposi's sarcoma-associated herpesvirus). The dissemination of herpes virus infection is influenced not only by the immune responses to the virus, but also by the likelihood that diffusion of the virus is modulated by HSPGs at the cell surface or within basement membranes. Thus, the potential implications for novel treatments using heparan sulfate mimetic are perceptible.
I hope that this Perspective series will stimulate the curiosity of the reader, with the intention of enticing new blood into the fruitful field of proteoglycan research, where a wealth of crops is waiting to be harvested.

\section{Acknowledgments}

I thank all the members of my laboratory, past and present, and Charles Reed for help with the artwork. I am deeply grateful to all the busy scientists who shared some of their valuable time with us to write such insightful and poignant Perspectives on the biology of heparan sulfate proteoglycans. The original work in the author's laboratory was supported by grants RO1 CA39481 and RO1 CA-47282 from the NIH and grant DAMD17-00-1-0663 from the Department of the Army.

1. Iozzo, R.V. 1998. Matrix proteoglycans: from molecular design to cellular function. Annu. Rev. Biochem. 67:609-652.

2. Iozzo, R.V. 2000. Proteoglycans: structure, biology and molecular interactions. Marcel Dekker Inc. New York, New York, USA. 422 pp.

3. Bernfield, M., et al. 1999. Functions of cell surface heparan sulfate proteoglycans. Annu. Rev. Biochem. 68:729-777.

4. Perrimon, N., and Bernfield, M. 2000. Specificities of heparan sulphate proteoglycans in developmental processes. Nature. 404:725-728.

5. Lander, A.D., and Selleck, S.B. 2000. The elusive functions of proteoglycans: in vivo veritas. J.Cell Biol. 148:227-232.

6. Lindahl, U., Kusche-Gullberg, M., and Kjellén, L. 1998. Regulated diversity of heparan sulfate. J. Biol. Chem. 273:24979-24982.

7. Sugahara, K., and Kitagawa, H. 2000. Recent advances in the study of the biosynthesis and functions of sulfated glycosaminoglycans. Curr. Opin. Struct. Biol. 10:518-527.

8. Nugent, M.A. 2000. Heparin sequencing brings structure to the function of complex oligosaccharides. Proc. Natl. Acad. Sci. USA. 97:10301-10303.

9. Turnbull, J., Powell, A., and Guimond, S. 2001. Heparan sulfate: decoding a dynamic multifunctional cell regulator. Trends Cell Biol. 11:75-82.

10. Keiser, N., Venkataraman, G., Shriver, Z., and Sasisekharan, R. 2001. Direct isolation and sequencing of specific protein-binding glycosaminoglycans. Nat. Med. 7:123-128.

11. Jenniskens, G.J., Oosterhof, A., Brandwijk, R., Veerkamp, J.H., and van Kuppevelt, T.H. 2000. Heparan sulfate heterogeneity in skeletal muscle basal lamina: demonstration by phage display-derived antibodies. J. Neurosci. 20:4099-4111.

12. Nicole, S., et al. 2000. Perlecan, the major proteoglycan of basement membranes, is altered in patients with Schwartz-Jampel syndrome (chondrodystrophic myotonia). Nat. Genet. 26:480-483.

13. Arikawa-Hirasawa, E., et al. 2001. Dyssegmental dysplasia, SilvermanHandmaker type, is caused by functional null mutations of the perlecan gene. Nat. Genet. 27:431-434.

14. Filmus, J., and Song, H.H. 2000. Glypicans. In Proteoglycans: structure, biology and molecular interactions. R.V. Iozzo, editor. Marcel Dekker Inc. New York, New York, USA. 161-176.

15. McCormick, C., et al. 1998. The putative tumour suppressor EXT1 alters the expression of cell-surface heparan sulfate. Nat. Genet. 19:158-161. 